\title{
Precise Predictions for Higgs-Boson Masses in the Next-to-Minimal Supersymmetric Standard Model (NMSSM)
}

\author{
Peter Drechsel* \\ Deutsches Elektronen-Synchrotron (DESY), Hamburg, Germany \\ E-mail: peter.drechseledesy.de
}

\section{Leonardo Galeta}

Instituto de Física de Cantabria (IFCA), Santander, Spain

E-mail: leodifca.unica.es

\section{Sven Heinemeyer}

Instituto de Física de Cantabria (IFCA), Santander, Spain

E-mail: heinemey@mail.cern.de

\section{Georg Weiglein}

Deutsches Elektronen-Synchrotron (DESY), Hamburg, Germany

E-mail: georg.weiglein@desy.de

\begin{abstract}
The NMSSM represents an elegant and well motivated description for the observed phenomenology in high energy physics. In this model a scalar singlet together with its superpartner is added to the Higgs sector of the Minimal Supersymmetric Standard Model (MSSM). In order to compare the NMSSM with experimental data at the same level of accuracy as the MSSM, precise predictions for Higgs-boson masses in the NMSSM are a necessity. This work will focus on the prediction for the Higgs masses in the NMSSM at one- and two-loop order obtained by Feynman diagrammatic methods. While the one-loop calculation is performed in the full NMSSM, the two-loop contributions to the Higgs-boson self-energies are approximated by their MSSM counterparts. It is shown that in this way the two-loop contributions are well approximated for a wide range of parameters. The results are exemplified for an example scenario genuine to the NMSSM.
\end{abstract}

The European Physical Society Conference on High Energy Physics

22-29 July 2015

Vienna, Austria

\footnotetext{
* Speaker.
} 


\section{Introduction}

The spectacular discovery of a boson with a mass around $125 \mathrm{GeV}$ by the ATLAS and CMS experiments [1,2] at CERN constitutes a milestone in the quest for understanding the physics of electroweak symmetry breaking. Any model describing electroweak physics needs to provide a state that can be identified with the observed signal. The measured mass value of the observed signal has already reached the level of a precision observable, with an experimental accuracy of better than $300 \mathrm{MeV}$ [8], and by itself provides an important test for the predictions of models of electroweak symmetry breaking. In order to fully exploit the precision of the experimental mass value for constraining the available parameter space of the considered models, the theoretical predictions should ultimately have an accuracy at the same level or better than the one of the experimental value.

In the case of the $C P$ conserving NMSSM (see e.g. [3,4] for reviews), which we assume throughout this work, the states in the NMSSM Higgs sector can be classified as three $C P$-even Higgs bosons, $H_{i}(i=1,2,3)$, two $C P$-odd Higgs bosons, $A_{j}(j=1,2)$, and the charged Higgs boson pair $H^{ \pm}$. In addition, the SUSY partner of the singlet Higgs (called the singlino) extends the neutralino sector (to a total of five neutralinos). In the NMSSM the lightest but also the second lightest $C P$-even neutral Higgs boson can be interpreted as the signal observed at $\approx 125 \mathrm{GeV}$. In order to improve the prediction for the Higgs masses in the NMSSM we present two-loop predictions that include the one-loop contributions in the full NMSSM and two-loop NMSSM contribution approximated by the two-loop MSSM contributions within a mixed on-shell and $\overline{\mathrm{DR}}$ renormalisation scheme, including the resummation of logarithms involving large SUSY masses. We will show that this approximation is valid for a wide range of parameters. The approximation itself and its quality will be briefly discussed in this work. The presented calculation will be included in the NMSSM version of the public tool FeynHiggs [6,7]. The preliminary version used to obtain the presented results is involved in comparison with tools dedicated to the NMSSM with a similar functionality, like NMSSMCALC [5].

\section{The Next-to-Minimal Supersymmetric Standard Model (NMSSM)}

The superpotential of the NMSSM for the third generation of fermions/sfermions reads

$$
W=Y_{t}\left(\hat{H}_{2} \cdot \hat{Q}_{3}\right) \hat{u}_{3}-Y_{d}\left(\hat{H}_{1} \cdot \hat{Q}_{3}\right) \hat{d}_{3}-Y_{\tau}\left(\hat{H}_{1} \cdot \hat{L}_{3}\right) \hat{e}_{3}+\lambda \hat{S}\left(\hat{H}_{2} \cdot \hat{H}_{1}\right)+\frac{1}{3} \kappa \hat{S}^{3},
$$

with the quark and lepton superfields $\hat{Q}_{3}, \hat{u}_{3}, \hat{d}_{3}, \hat{L}_{3}, \hat{e}_{3}$ and the Higgs superfields $\hat{H}_{1}, \hat{H}_{s}, \hat{S}$. The $S U(2)_{\mathrm{L}}$-invariant product is denoted by a dot. The Higgs singlet and doublets are decomposed into $C P$-even and $C P$-odd neutral scalars $\phi_{i}$ and $\chi_{i}$, and charged states $\phi_{i}^{ \pm}$,

$$
H_{1}=\left(\begin{array}{c}
v_{1}+\frac{1}{\sqrt{2}}\left(\phi_{1}-i \chi_{1}\right) \\
-\phi_{1}^{-}
\end{array}\right), \quad H_{2}=\left(\begin{array}{c}
\phi_{2}^{+} \\
v_{2}+\frac{1}{\sqrt{2}}\left(\phi_{2}+i \chi_{2}\right)
\end{array}\right), \quad S=v_{s}+\frac{1}{\sqrt{2}}\left(\phi_{s}+i \chi_{s}\right),
$$

with the real vacuum expectation values for the doublet- and the singlet-fields, $v_{\{1,2\}}$ and $v_{s}$. Since $\hat{S}$ transforms as a singlet, the $D$-terms remain identical to the ones of the MSSM. The parameters $Y_{f}$ denote the Yukawa couplings of the SM fermions. The parameters $\lambda$ and $\kappa$ are genuine to the 
NMSSM and can be chosen freely. However, in order to keep the validity of perturbation theory up to the GUT scale [3], $\lambda$ and $\kappa$ are bound from above by

$$
\lambda^{2}+\kappa^{2} \lesssim 0.5
$$

so that $\lambda \lesssim 0.7$, where the largest values are only allowed for vanishing $\kappa$. Thus $Y_{t} \approx 1>\lambda$ is ensured by this constraint. Throughout this work this constraint will be assumed for the NMSSM.

The SUSY breaking terms for the trilinear breaking parameters $A$ read

$$
\mathscr{L}_{\text {soft }} \supset Y_{t} A_{t} \tilde{t}_{L} H_{2} \tilde{t}_{R}+Y_{b} A_{b} \tilde{b}_{L} H_{1} \tilde{b}_{R}+Y_{\tau} A_{\tau} \tilde{\tau}_{L} H_{1} \tilde{\tau}_{R}++\lambda A_{\lambda} S H_{2} H_{1}+\frac{1}{3} \kappa A_{\kappa} S^{3}+\text { h.c. . }
$$

\section{Pole Mass Determination}

The masses of the Higgs bosons are obtained using1 diagrammatic methods and determining1 the complex pole of the full propagator. For a scalar field $\phi$ this reads

$$
\Delta^{-1}\left(k^{2}\right)=\mathrm{i}\left[k^{2} \mathbb{1}-\mathscr{M}_{\phi \phi}+\hat{\Sigma}_{\phi \phi}\left(k^{2}\right)\right],\left.\quad \Delta^{-1}\left(k^{2}\right)\right|_{k^{2}=m_{\phi}^{2}-\mathrm{i} \Gamma_{\phi} m_{\phi}} \stackrel{!}{=} 0
$$

The complex pole consists of the mass $m_{\phi}$ and the total width $\Gamma_{\phi}$. The renormalised self-energy $\hat{\Sigma}_{\phi \phi}$ is evaluated by taking into account the full contributions from the NMSSM at one-loop order and as an approximation the dominant contribution from the MSSM at two-loop order,

$$
\left.\hat{\Sigma}_{\phi \phi}\left(k^{2}\right) \approx \hat{\Sigma}_{\phi \phi}^{(1 \mathrm{~L})}\left(k^{2}\right)\right|^{\mathrm{NMSSM}}+\left.\hat{\Sigma}_{\phi \phi}^{(2 \mathrm{~L})}\left(k^{2}\right)\right|_{k^{2}=0} ^{\mathrm{MSSM}}
$$

It includes at two-loop order the MSSM contributions of $\mathscr{O}\left(\alpha_{s} \alpha_{t}, \alpha_{s} \alpha_{b}, \alpha_{t}^{2}, \alpha_{t} \alpha_{b}\right)$ and resummed large logarithms induced by heavy SUSY particles. These contributions are taken directly from the MSSM version of FeynHiggs. The included contributions from the quark and squark sectors represent the numerically leading contributions as known in the MSSM calculated for vanishing external momentum.

\section{Employed Approximation}

The self energy $\Sigma_{\phi \phi}$ for the $C P$-even Higgs fields in the NMSSM is a $3 \times 3$ matrix. For the contributions from the top and stops its entries can be classified by the dominant coupling constants. At one-loop order this classification separates the self energy into an MSSM-like $2 \times 2$ sub-matrix and corrections that are genuine to the NMSSM,

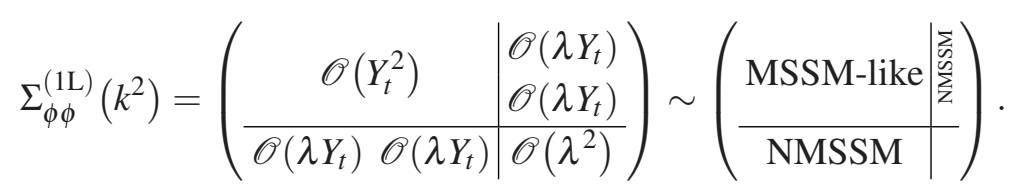

In the regime where the NMSSM stays perturbative up to the GUT scale $\lambda<Y_{t}$ holds, so that the genuine NMSSM corrections are expected to be suppressed compared to the MSSM-like corrections. This suppression also applies to the contributions from top/stops and a gluon/gluino at 
two-loop order. Here the leading contributions consist of a one-loop topology with an inserted gluon or gluino propagator, like

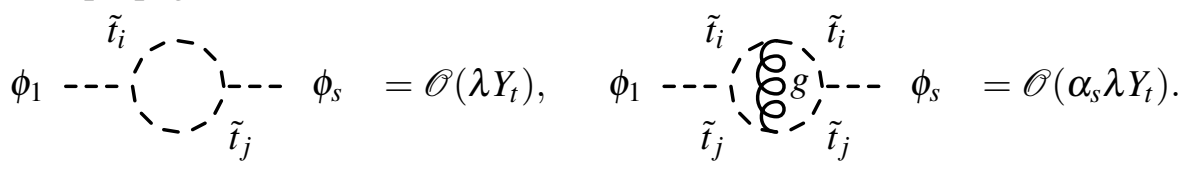

The genuine two-loop NMSSM corrections are thus neglected for the presented results,

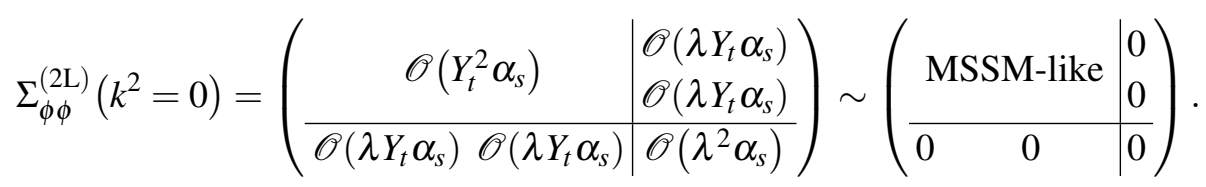

Since the structure of the dominant contributions at the two-loop level of $\mathscr{O}\left(Y_{t}^{2} \alpha_{s}\right)$ in comparison with the genuine NMSSM contributions of $\mathscr{O}\left(\lambda Y_{t} \alpha_{s}, \lambda^{2} \alpha_{s}\right)$ is very similar to the corresponding contributions at one-loop order, testing the quality of the one-loop approximation, where the genuine NMSSM corrections are neglected, but known, also provides a test for the quality of the two-loop approximation. For the two-loop corrections beyond $\mathscr{O}\left(Y_{t}^{2} \alpha_{s}\right)$ the approximation by their MSSM counterparts is employed.

\section{Renormalisation Conditions}

At one-loop order the parameters from the Higgs potential and the gauge sector have to be renormalised. We choose to renormalise the tadpoles to zero at all orders of perturbation theory. Rather than choosing the gauge couplings and the soft-breaking parameter $A_{\lambda}$ as input parameters, the gauge-boson masses $M_{W}, M_{Z}$ and charged Higgs mass $M_{H^{ \pm}}$were chosen as independent parameters. The following renormalisation schemes are chosen for the parameters entering at one-loop order

$$
\text { on-shell: } M_{W}, M_{Z}, M_{H^{ \pm}} \quad \overline{\mathrm{DR}}: \lambda, \kappa, A_{\kappa}, \tan \beta, \mu_{\mathrm{eff}}, v=\sqrt{v_{1}^{2}+v_{2}^{2}} .
$$

Since the vacuum expectation-value $v$ is directly related to the electromagnetic coupling constant charge $e$ a reparametrisation for the electric charge $e$ is necessary in order to use a given numerical value for the electromagnetic coupling constant. In the following the value for $e$ derived from Fermi's constant is used, which is the parametrisation that was chosen for the results that are implemented in FeynHiggs.

\section{Results: A genuine NMSSM Scenario}

The sample scenario investigated here is defined by the parameters in the Higgs sector given in eq. (6.1a), while $\lambda$ is varied. For values $\lambda \gtrsim 0.32$ the mass of the lightest Higgs state becomes tachyonic at tree-level. The analyses will therefore be restricted to values of $\lambda \lesssim 0.32$. The parameters entering at higher order are chosen as given in eq. (6.1b).

$$
\begin{aligned}
& M_{H^{ \pm}}=1000 \mathrm{GeV}, \mu_{\mathrm{eff}}=125 \mathrm{GeV}, A_{\kappa}=-300 \mathrm{GeV}, \kappa=0.2, \tan \beta=8, \\
& M_{\tilde{q}}=1500 \mathrm{GeV}, M_{\tilde{l}}=200 \mathrm{GeV}, M_{1}=\frac{5}{3} \frac{s_{\mathrm{w}}^{2}}{c_{\mathrm{w}}^{2}} M_{2} \approx 143 \mathrm{GeV}, M_{2}=300 \mathrm{GeV}, M_{3}=1500 \mathrm{GeV} \\
& A_{t}=-2000 \mathrm{GeV}, A_{\tau}=A_{b}=-1500 \mathrm{GeV}, A_{q}=-1500 \mathrm{GeV}, A_{l}=-100 \mathrm{GeV} .
\end{aligned}
$$


The parameters $M_{\tilde{f}}$ and $A_{\tilde{f}}$ specify the universal sfermion-mass and trilinear breaking-parameters, while $M_{\{1,2,3\}}$ are the gaugino mass breaking parameters for $U(1)_{Y}, S U(2)_{\mathrm{L}}$ and $S U(3)_{\mathrm{c}}$. All stopsector parameters are understood as on-shell parameters [9].

The masses of the two lighter Higgs fields in this genuine NMSSM scenario are given in fig. 1 . At two-loop order the lightest state is singlet-like for values $\lambda \gtrsim 0.23$, while the second lightest state in this parameter region is SM-like with a mass of $\approx 125 \mathrm{GeV}$. For values $\lambda \lesssim 0.23$ the lightest state is doublet-like $\approx 125 \mathrm{GeV}$ and the second lightest is singlet-like. The heaviest $C P$-even Higgs field remains doublet-like with a mass of for the shown parameters with a mass of $\approx 1 \mathrm{TeV}$. The scenario has been tested with HiggsBounds 4.1 .3 [10] to ensure its experimental viability.
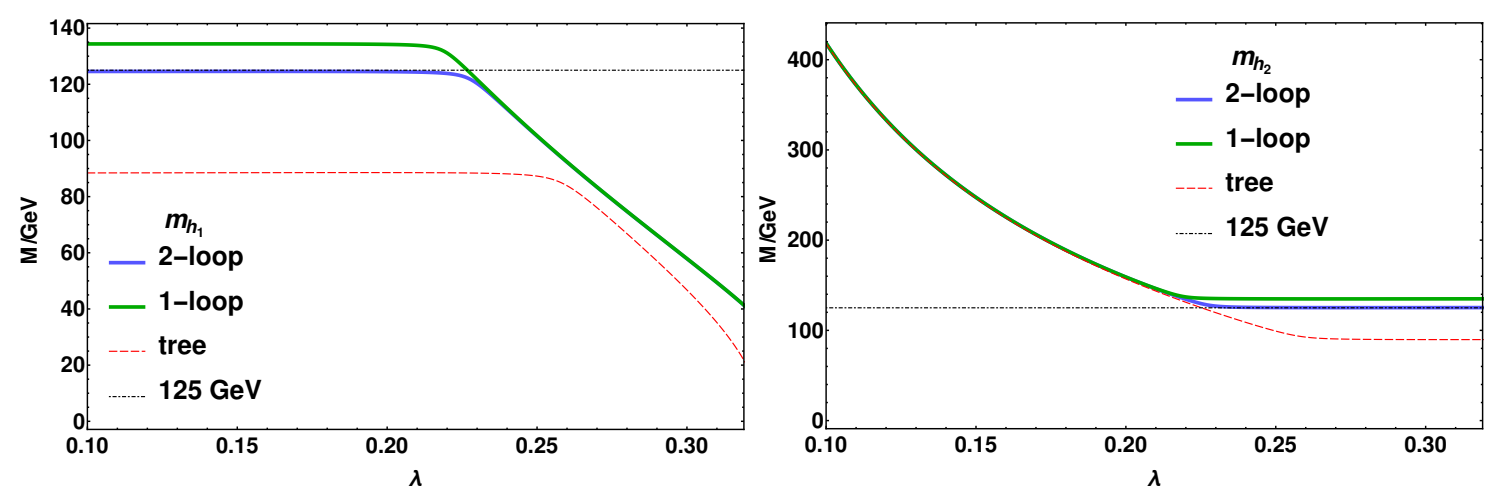

Figure 1: Masses of the two lighter $C P$-even Higgs fields as a function of $\lambda$ at tree-, one-loop and two-loop level.

\section{Validity of Approximation}

The masses of the two lighter $C P$-even Higgs fields are studied at one-loop order for the dominant contributions from top and stops in fig. 2. In the left plot the absolute difference between the results in- and excluding the genuine NMSSM corrections is shown. The impact of the genuine corrections is very small with less than $100 \mathrm{MeV}$ for $\lambda \lesssim 0.25$, while the contribution to $m_{h_{1}}$ sharply increases for larger values of $\lambda$. In the right plot the absolute difference between the result including only the top/stop contributions of $\mathscr{O}\left(Y_{t}^{2}, \lambda Y_{t}, \lambda^{2}\right)$, denoted by $m_{t}^{4}$, and the full one-loop mass prediction is shown by solid lines. The dashed lines show the same difference between the result including top/stop contributions plus contributions from the Higgs/higgsino/gauge-boson/gaugino sectors including their superpartners, denoted by $m_{t}^{4}+\mathrm{HG}$, and the full one-loop mass predictions.

For the largest values of $\lambda \approx 0.32$ one-loop corrections at from the Higgs/higgsino/gaugeboson/gaugino sectors amount to $\approx 20 \mathrm{GeV}$, roughly the same size as the leading top/stop contributions. In this region, where the corrections stop corrections of $\mathscr{O}\left(\lambda Y_{t}, \lambda^{2}\right)$ gain numerical impact, the corrections from the Higgs sector become more important. The uncertainty from excluding the genuine NMSSM-corrections is far less severe then the exclusion of the Higgs and gauge sector contributions.

\section{Conclusion}

The Higgs-mass prediction including the full one-loop calculation in the NMSSM and all avail- 

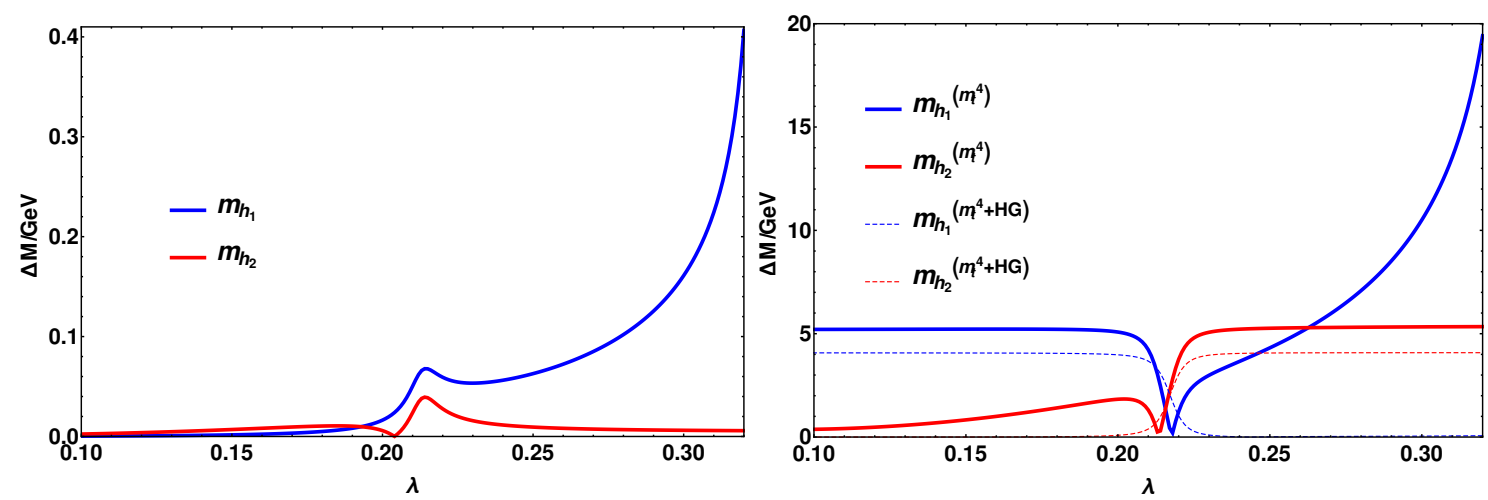

Figure 2: Difference between one-loop mass-predictions with different contributions for the two lighter Higgs fields. Left: Absolute difference between the results including leading top/stop corrections with and without the corrections of $\mathscr{O}\left(\lambda Y_{t}, \lambda^{2}\right)$. Right: Absolute difference between the result including the top/stop contributions of $\mathscr{O}\left(Y_{t}^{2}, \lambda Y_{t}, \lambda^{2}\right)$, denoted by $m_{t}^{4}$, and the full one-loop mass prediction (solid lines). Absolute difference between the result including top/stop contributions plus contributions from the Higgs/higgsino/gauge-boson/gaugino sectors including their superpartners, denoted by $m_{t}^{4}+\mathrm{HG}$, and the full one-loop mass predictions (dashed lines).

able two-loop contributions from the MSSM have been presented. Its validity in the perturbative regime for $\lambda$ and $\kappa$ has been confirmed.

\section{References}

[1] G. Aad et al. [ATLAS Collaboration], Phys. Lett. B 716 (2013) 1 [arXiv:1207.7214 [hep-ex]].

[2] S. Chatrchyan et al. [CMS Collaboration], Phys. Lett. B 716 (2013) 30 [arXiv:1207.7235 [hep-ex]].

[3] U. Ellwanger, C. Hugonie and A. M. Teixeira, Phys. Rept. 496 (2010) 1 [arXiv:0910.1785 [hep-ph]].

[4] M. Maniatis, Int. J. Mod. Phys. A 25 (2010) 3505 [arXiv:0906.0777 [hep-ph]].

[5] J. Baglio, R. Gröber, M. Mühlleitner, D. T. Nhung, H. Rzehak, M. Spira, J. Streicher and K. Walz, Comput. Phys. Commun. 185 (2014) 12, 3372 [arXiv:1312.4788 [hep-ph]].

[6] G. Degrassi, S. Heinemeyer, W. Hollik, P. Slavich and G. Weiglein, Eur. Phys. J. C 28 (2003) 133 [hep-ph/0212020].

[7] T. Hahn, S. Heinemeyer, W. Hollik, H. Rzehak and G. Weiglein, Phys. Rev. Lett. 112 (2014) 14, 141801 [arXiv:1312.4937 [hep-ph]].

[8] G. Aad et al. [ATLAS and CMS Collaborations], Phys. Rev. Lett. 114 (2015) 191803 [arXiv:1503.07589 [hep-ex]].

[9] S. Heinemeyer, W. Hollik and G. Weiglein, Eur. Phys. J. C 9 (1999) 343 [hep-ph/9812472].

[10] P. Bechtle, O. Brein, S. Heinemeyer, O. StÃěl, T. Stefaniak, G. Weiglein and K. E. Williams, Eur. Phys. J. C 74 (2014) 3, 2693 [arXiv:1311.0055 [hep-ph]]. 